\title{
Insistieren oder sich auf Gottes Willen ver- lassen? Die Gebetsparänese im Matthäus- und im Lukasevangelium
}

Lorenzo Scornaienchi

\section{Einleitung: zwei Auffassungen vom Gebet}

Wenn man die Auffassungen vom Gebet im Matthäus- und im Lukasevangelium vergleicht, stellt man einen wesentlichen Unterschied fest. Im Lukasevangelium liegt der Akzent auf der Beharrlichkeit des Betenden, auf seinem kontinuierlichen Insistieren, im Matthäusevangelium wird hingegen eine andere Haltung propagiert: Man soll im Gebet nicht viele Worte verwenden, man soll beim Beten auf keinen Fall Worte anhäufen, weil Gott schon vorher weiss, was für den Menschen nötig ist (Mt 6,6-9). Dieser Kontrast bestätigt sich auch im Blick auf das Vaterunser: Zwar ist die matthäische Version länger als die lukanische. Während aber im Matthäusevangelium das Vaterunser als Vorbild des wesentlichen und knappen Betens vorgestellt wird (und im Vergleich mit den gängigen Gebeten der Zeit ist es tatsächlich ein knappes Gebet), begegnet es in Lk 11 in einem Kontext, in dem - vielleicht wegen seines konkreten Bezugs auf die Bitte um das Brot - die Bedeutung des Beharrens im Beten im Vordergrund steht. Es stellt sich die Frage: Wie kann man diese unterschiedlichen Auffassungen der beiden Evangelien zum Gebet erklären? Handelt es sich einfach um verschiedene Akzentuierungen oder drücken sich in diesen Unterschieden völlig andere Konzeptionen des Wesens des Gebets aus? Solche Fragen können auch behilflich sein, um die Bedeutung des Gebets in der frühen Kirche $\mathrm{zu}$ verstehen.

O. Cullmann versucht in seinem Buch über das Gebet im Neuen Testament, die Perspektiven der beiden Evangelien durch eine paradoxe Aussage zu verbinden: "Gott braucht nicht, aber er will das Gebet der Menschen «" ${ }^{1}$ das Gebet ist nicht notwendig, aber es ist erwünscht. Er entnimmt diesen Gedanken dem Lied Paul Gerhardts "Befiehl du deine Wege«, und zwar insbesondere den Worten:

1 O. Cullmann, Das Gebet im Neuen Testament. Zugleich Versuch einer vom Neuen Testament aus zu erteilenden Antwort auf heutige Fragen, Tübingen 1994, 29. 
»Mit Sorgen und mit Grämen

und selbsteigener Pein

lässt Gott sich gar nicht nehmen;

Es muss erbeten sein «. ${ }^{2}$

Obwohl Cullmann das Müssen des Betens im letzten Vers Gerhardts betont, liegt die grundlegende Vorstellung des Lieds im Gedanken, dass Gott die Geschicke trotz jeden Widerstrebens der Menschen ihrem Ziel zuführt. Dies kommt klar in folgenden Worten zu Sprache:

»Und was du dann erlesen,

das treibst du starker Held,

und bringst zum Stand und Wesen,

was deinem Rath gefällt«. ${ }^{3}$

Die Synthese der beiden Perspektiven geschieht nach Cullmann durch das Paradox Gott braucht keine Gebete, aber er will sie. Er erinnert daran, dass die Beharrlichkeit beim Beten auf die Radikalität Jesu zurückgeht: »Den unbedingten Willen Gottes, dass wir zu ihm beten, hat Jesus in seiner ganzen Radikalität erfasst. Daher die wiederholte, so eindringliche Aufforderung zur Beharrlichkeit beim Beten. Die Notwendigkeit dieser Beharrlichkeit entspricht dem göttlichen Willen. ${ }^{4}$ Cullmanns Lösung des Problems der verschiedenen Ansichten zum Gebet ist also, eine paradoxe Systematisierung zu unternehmen und alles auf die Radikalität Jesu zurückzuführen. Diese Erklärung ist aber nicht ausreichend, um die Komplexität der beiden Evangelien zu fassen. Vielmehr ist es notwendig, die beiden Perspektiven des Insistierens und des Vertrauens auf Gottes Vorsehung zuerst je für sich zu verstehen und dann zu hinterfragen, ob eine Synthese überhaupt möglich ist.

Bevor wir damit beginnen, möchte ich einige methodische Vorbemerkungen anbringen: (1.) Da das Thema Gebet sehr weit ist, konzentriert sich meine Untersuchung auf konzeptionelle Texte über das Gebet, die aus dem Sondergut der beiden Evangelien stammen, nämlich die Paränese über das Gebet in Mt 6,6-9 und die Paränese durch die lukanischen Gleichnisse in Lk 11 und 18. Das Sondergut ist deswegen interessant, weil es das Proprium jedes Evangeliums darstellt und damit die jeweilige eigentümliche Ansicht zum Gebet klarer hervorgehoben wird. Beide Evangelien enthalten verschiede-

2 Cullmann, Das Gebet im Neuen Testament, 31.

3 Paulus Gerhardts geistliche Lieder (Bd. 1), hg. v. K. Gerok, Leipzig ${ }^{3} 1883$, 230 , 3. Strophe. Der gleiche Gedanke wird auch in der 5. und in den folgenden Strophen betont.

4 Cullmann, Das Gebet im Neuen Testament, 30. 
ne Episoden, die das Gebet betreffen. Es geht vor allem um Gebete oder Gebetshandlungen Jesu. Diese Texte sind für unsere Untersuchung wenig interessant, weil sie aus dem gleichen traditionellen Material stammen. (2.) Dieser Vergleich der matthäischen und der lukanischen Auffassung zum Gebet will außerdem auf eine Lücke in der Literatur zum Gebet in den Evangelien hinweisen. Die meisten Monographien befassen sich mit der Gebetskonzeption des lukanischen Doppelwerkes ${ }^{5}$ und nicht mit der matthäischen Vorstellung des Gebets. Diejenigen Forscher, die das Matthäusevangelium behandeln, konzentrieren sich dagegen vor allem auf die Untersuchung des Vaterunsers. (3.) Eine letzte Präzisierung betrifft die lexikalische und semantische Erforschung des Themas. Die griechischen Termini, die das Gebet bezeichnen, sind zahlreich. ${ }^{6}$ Die zu untersuchenden Texte verwenden das Verb $\pi \rho 0 \sigma \varepsilon v ́ \chi \varepsilon \sigma \theta \alpha$, das im Neuen Testament am meisten benutzt wird. Die Paränesen der beiden Evangelien befassen sich mit dem Bittgebet. Eine Unterscheidung der vielen Formen des Gebets ist in dieser Gegenüberstellung nicht nützlich.

\section{Das Gebet nach Matthäus: Wenige Worte im persönlichen Gebet}

Die matthäische Gebetsparänese ist fast ausschließlich in der Bergpredigt eingebettet. ${ }^{7}$ Jesus diskutiert dort drei Handlungen der damaligen Frömmigkeit: das Beten, das Almosengeben und das Fasten.

\footnotetext{
5 Zahlreich sind die Untersuchungen zum Gebet im Lukasevangelium und in der Apostelgeschichte. Ich nenne hier einige wichtige Beispiele:W. Ott, Gebet und Heil. Die Bedeutung der Gebetsparänese in der lukanischen Theologie, StANT 12, München 1965; L. Feldkämper, Der betende Jesus als Heilsmittler nach Lukas, Veröffentlichungen des Missionspriesterseminars St. Augustin bei Bonn, 29, St. Augustin 1978; S.F. Plymale, The Prayer in Luke-Acts, American University Studies - Theology and Religion 118, New York u.a. 1991; N. Förster, Das gemeinschaftliche Gebet in der Sicht des Lukas, Biblical Tools and Studies 4, Leuven u.a. 2007; K. Pfremmer De Long, Surprised by God. Praise Response in the Narrative of Luke-Acts, BZNW 166, Berlin/New York 2009; G.O. Holmas, Prayer and Vindication in Luke-Acts. The Theme of Prayer within the Context of the Legitimating and Edifying Objective of the Lukan Narrative, Library of the New Testament Studies 433, London/New York 2011. Detaillierte Forschungsberichte finden sich bei D.M. Crump, Jesus the Intercessor. Prayer and Christology in Luke-Acts, WUNT II/49, Tübingen 1992, 2-11; und bei Förster, Das gemeinschaftliche Gebet in der Sicht des Lukas, 2-21.

6 Vgl. Holmas, Prayer and Vindication in Luke-Acts, 29f.

7 Wenn man der Hypothese von U. Luz (Das Evangelium nach Matthäus, I. Mt 1-7, EKK I/1, Düsseldorf/ Zürich ${ }^{5}$ 2002, 254) zustimmt, nach der das Vaterunser das Zentrum der Bergpredigt darstellt, spielt dieses Gebet und die Gebetsparänese für die
} 
Obwohl sich diese Handlungen direkt auf die jüdische Frömmigkeit und Praxis beziehen, lassen sie sich ebenfalls auf andere Religionen anwenden. Jede Religion beschäftigt sich mit Fasten, mit Beten und mit Wohltaten. Das Besondere in der Auffassung Jesu ist die Idee, dass die wahre Frömmigkeit einen persönlichen und intimen Charakter hat, der zu jedem äusserlichen Ausdruck im Gegensatz steht. Was wir mit dem Begriff „Frömmigkeit» bezeichnen können, wird im Text des Matthäusevangeliums mit dem zentralen Terminus

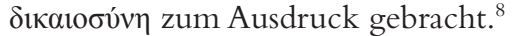

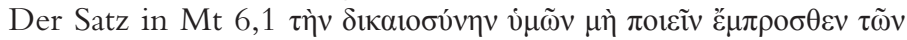

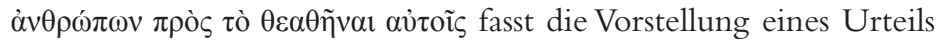
über die Frömmigkeit zusammen. Der Zweck der Mahnungen ist, eine innerliche Frömmigkeit vor Gott zu suchen. Jeder Versuch der Externalisierung oder Selbstdarstellung steht sonst unter der Gefahr,

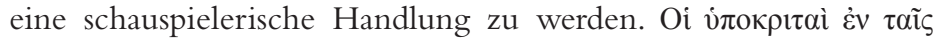

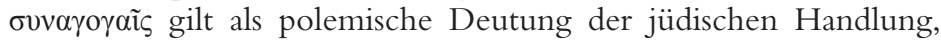
weil der bestimmte Artikel auf eine besondere Gruppierung hinweist. Sie lässt sich aber auch auf andere Formen externer Darstellung der Frömmigkeit anwenden. Die Basis für die christliche Frömmigkeit ist die Innerlichkeit seines Ausdrucks und die Exklusivität des Verhältnisses zu Gott, weil Gott ins Innere des Menschen sieht und keine Vermittlung durch Externalisierung braucht. Wenn man in die Äußerlichkeit investiert, befindet man sich bereits in der Dimension des Urteils Gottes, der von solchen Handlungen nicht erreicht wird. Das Einzige, was man erreicht, was im Text mit "Lohn « bezeichnet wird, ist der Vollzug einer Selbstdarstellung. Wie ein Refrain lautet

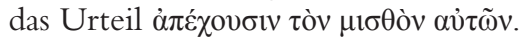

In Fall des Gebets werden zwei Elemente unterschieden: der Ort, wo man betet, und die Art wie man betet. Mt 6,5-6 und 6,7-8 sind parallel aufgebaut und präsentieren zwei Oppositionen.Die vं

Bergpredigt eine wichtige Rolle. Das Gebet wird dann zum Höhepunkt des neuen ethischen Entwurfs. Luz erklärt diese zentrale Stelle weniger konzeptionell und inhaltlich als reine 'geometrischeく Mitte der Kapitel 5-7 des Matthäusevangeliums. Eine weitere Vorstellung sieht im Vaterunser eine Synthese aller Themen der Bergpredigt, vgl. W. Fenske, "und wenn ihr betet ... (Mt 6,5). Gebete in der zwischenmenschlichen Kommunikation der Antike als Ausdruck der Frömmigkeit, StUNT 21, 63-64. Die These lautet wie folgt: Die ersten drei Bitten des Vaterunsers entsprechen den Macharismen (Mt 5,1-12) und den neuen Geboten (Mt 5,13-6,18), die letzten vier Bitten dem Nichtbesorgtsein um die Nahrung (Mt 6,19-34), dem Nicht-Richten (Mt 7,1-5), der Entweihung des Heiligen (Mt 7,6), dem Bitten (Mt 7,7-11).

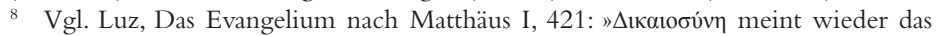
menschliche Handeln, wie es Gott, der himmlische Vater, will. Es entspricht sachlich dem, was wir heute »Frömmigkeit» nennen würden.» 
wählen einen Ort zum Beten, wo viele Menschen versammelt sind: die Synagogen und die Ecken der Plätze. Sie wählen diese Orte, damit viele Menschen sie sehen können. Auf dieser Gegebenheit wird die Opposition aufgebaut. Die Rezipienten des Textes müssen einen ganz anderen Ort zum Beten wählen, einen Ort, das $\tau \alpha \mu \varepsilon \tau_{0} v^{9}$, der im extremen Gegensatz zu diesen öffentlichen Räumen steht. Dieses Wort bezeichnet ein kleines Zimmer im Haus, wo meistens Lebensmittel aufbewahrt wurden. ${ }^{10}$ Die Öffentlichkeit der Synagoge und des Platzes wird dem Privaten des "Kämmerchens" gegenübergestellt. Dazu muss der christliche Betende noch die Tür verriegeln. Sein Beten wird als nur an Gott gerichtetes Beten verstanden, das keinen anderen Menschen betreffen soll. Die Begründung dieser Wahl eines intimen Ortes liegt in der Tatsache, dass im Gebet das Gespräch mit Gott gesucht wird und dass Gott das Verborgene sieht, ohne sich von Äußerlichkeiten beeinflussen zu lassen.

Die zweite Opposition betrifft die Art des Betens. Das negative Beispiel sind die Heiden, die viele Worte zum Beten verwenden.

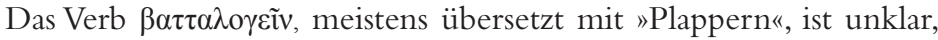
weil es praktisch nur an dieser Stelle vorkommt. ${ }^{11}$ Es wird meistens

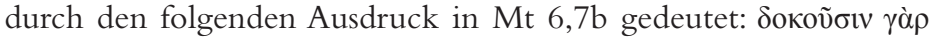

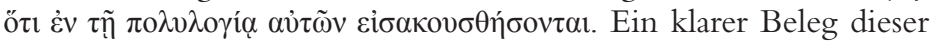

\footnotetext{
9 Die Benennung eines Zimmers im Haus, wo das Gebet geschehen soll, ist ein Hinweis, dass in der matthäischen Rezeption das Gebet in Verbindung mit einem sesshaften Leben steht und nicht mehr der Erfahrung einer Gruppe von Wanderpredigern entspricht. Es geht daher um eine Ortsgemeinde, die diese Unterweisung rezipieren soll.

${ }^{10}$ K.L. Grimm, Lexicon Graeco-Latinum in Novi Testamenti, Leipzig ${ }^{3} 1888$, 426, unterscheidet folgende Bedeutungen des Terminus: »1) cella penaria, promptuarium (Vorrathskammer ) ... 2) conclave, maxime interius; penetrale«. Ähnlich auch W. Baur, Griechisch-Deutsches Wörterbuch zu den Schriften des Neuen Testaments und der übrigen urchristlichen Literatur, Berlin $\left.{ }^{4} 1952,1460, » 1\right)$ Vorrathskammer, [... 2) d. innerste Gemach".

${ }^{11}$ Baur, Wörterbuch, 250, nennt zwei weitere Belege bei der Vita Aesopi (ed. Westermann 1850, 19, p. 47,1) und Simplicius, Comm. in Epict. Enchr. 37. Der Codex

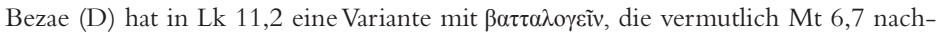
gebildet ist. Nach Baur ist das Verb vielleicht eine "hybride Bildung" des aramäischen Ausdrucks אתלטב רמא "leer sprechen«. Nach Grimm, Lexicon, 68, ist das Verb eher

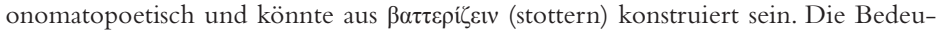
tung wäre dann »undeutlich sprechen«, was zu dem paganen wiederholenden Flehen gut passen würde. Im undeutlichen und unverständlichen Sprechen liegt auch der Ursprung des Wortes $\beta \alpha ́ \rho \beta \alpha \rho o \zeta$. Luz, Das Evangelium nach Matthäus I, teilt diese

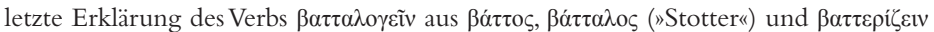
("stottern«): S. 430: "Gedacht ist wohl an die Repetition in sich sinnloser Silben. In-

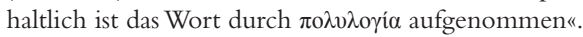


Praxis ist bei Titus Livius zu finden, der die Rolle der Frauen ${ }^{12}$ vor der Schlacht gegen Karthago beschreibt: Sie können nichts anders tun als Beten ${ }^{13}$ und ermüden damit durch wiederholtes Flehen und durch Opfergaben die Götter ("fatigare deos»). ${ }^{14}$ Kritisch dazu äußert Seneca, der in einem seiner moralischen Briefe an Lucilius schreibt, Selbstvertrauen sei die richtige Einstellung, um die Schwierigkeiten im Leben zu überwinden, und sogar der Garant für ein gutes Leben. ${ }^{15}$ Das setzt voraus, dass der so erfahrene Freund sich von der religiösen Praxis der Eltern löst. Ihm als erfolgreichen und bekannten Mann sei es nicht angemessen, die Götter mürbe zu machen. Er könne durch sein eigenes Tun glücklich werden. ${ }^{16}$ Seneca kritisiert das Beten tout court, das als ein Ersatz für das eigene Handeln verstanden wird. Eine kritische Einstellung zum Gebrauch von vielen Worten im Gebet ist bereits im Alten Testament enthalten, in Jes 1,15 und vor allem in der Auseinandersetzung des Elias mit den Priestern des Baal in 1Kön 18,27. Die Begründung, auf das Plappern und auf die Vielsprecherei zu verzichten, liegt in derVorsehung Gottes. Er weiß nämlich schon alles, was die Menschen benötigen, bevor sie im Gebet darum bitten. Die Folge dieser radikalen Auffassung ist aber nicht der Verzicht auf jegliches Beten, sondern die Anwendung desjenigen Gebets, das das Wesentliche enthält, das Vaterunser.

\subsection{Der Verzicht aufs Beten: das Beispiel von Maximus von Tyrus}

Eine extreme Interpretation der matthäischen Auffassung des Gebets könnte dazu führen, dass man ganz auf das Beten verzichtet. Wenn Gott alles schon vorher weiß, bevor man ein Gebet spricht, was hat es dann noch für einen Sinn zu beten? Beten scheint in dieser Hinsicht nicht die Vorbedingung dafür zu sein, dass man diejenigen Dinge bekommt, die man benötigt. Das Matthäusevangelium vertritt nicht diese extreme Position, sondern benutzt die hyperbolische

\footnotetext{
12 Vgl. J. Rüppke, Domi militiae: Die religiöse Konstruktion des Krieges in Rom, Stuttgart 1990, 66. Die Frauen konnten nicht am Krieg teilnehmen. Ihnen blieben die religiöse Praxis und die Unterstützung durch Beten übrig.

${ }^{13}$ Liv. 27,50,5 "matronae, qui nihil in ipsis opis erat, in preces obtestationesque versae, per omnia delubra vagae suppliciis votis fatigare deos".

${ }^{14}$ Der Ausdruck kann in verschiedenen Varianten vorkommen, z.B. "fatigare coelum" Phaedr. 4,12,24; "fatigare numina" Lucr. 4,1239, "fatigare Vestam» Hor. 1,2,26ff.

${ }^{15}$ Sen. Ep 31,3 "unum bonum est, quod beatae vitae causa et firmamentum est, sibi fidere".

${ }^{16}$ Sen. Ep. 31,5 "et in totum iam per maxima acto viro turpe est etiam nunc deos fatigare. Quid votis opus est? Fac te ipse felicem«.
} 
Argumentation, um sich auf das Wesentliche zu konzentrieren. Die Lösung ist die Knappheit und die Wesentlichkeit des Vaterunsers.

Die extreme Position des Verzichts auf das Gebet vertritt der Philosoph und Rhetoriker Maximus von Tyrus in seiner kurzen Abhandlung über die Nützlichkeit des Gebetes. ${ }^{17}$ Es lohnt sich, diese Position näher anzuschauen, obwohl sie chronologisch einige Jahrzehnte nach der Abfassung des Matthäusevangeliums zu datieren ist. Maximus wirkte nämlich Ende des zweiten Jahrhunderts nach Christus unter dem Kaiser Commodus. Er konzentriert sich auf das Bittgebet, in dem die meisten Menschen von den Göttern Reichtum und Erfolg verlangen. Er aber macht sich nicht nur über diese Extremfälle lustig, sondern über alle Arten von Bittgebet. Maximus bezieht sich auf die Geschichte des phrygischen Königs Midas, dem auf seinen Wunsch hin alle seine Äcker in Gold verwandelt wurden. Als der König dann merkte, dass somit auch alle seine Früchte aus Gold waren und er nichts mehr essen konnte, wollte er alles rückgängig machen. Das blieb aber ohne Erfolg.

Um die Sinnlosigkeit des Bittgebets zu zeigen, unterscheidet er in seiner Abhandlung, welche möglichen Ursachen ein Geschehen bestimmen. Einige Ereignisse kommen von derVorsehung ( $\pi \rho \operatorname{vov} 1 \alpha)$

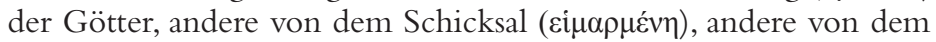

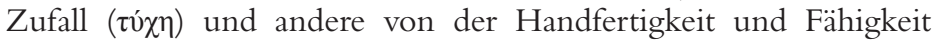

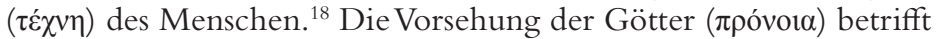
das Ganze und nicht den Einzelnen. Das Beten eines winzigen Teils des Universums, z.B. des einzelnen Menschen, für sein Nützliches hat nach Maximus wenig Sinn. Es gleicht dem Gebet eines kranken Körpergliedes, das den Arzt bittet, nicht weggeschnitten zu werden, obwohl dies die Voraussetzung für die Genesung des Ganzen wäre. Hier kommt die auch sonst in der hellenistischen Ethik und Politik verbreitete Metapher des Leibes zum Zug, die die Notwendigkeit des Ganzen über die Interessen des Einzelnen stellt. ${ }^{19}$ Es sei absurd,

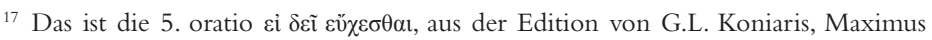
Tyrius Philosophumena, $\delta 1 \alpha \lambda \varepsilon \dot{\varepsilon} \xi \varepsilon \varsigma$, , Texte und Kommentare 17, Berlin/New York 1995. ${ }_{18}$ Max. Tyr 5,4a.

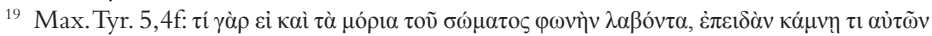

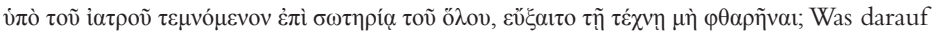
folgt ist ein Tadel von Asklepius selbst, der das kranke Glied daran erinnert, dass es für die Rettung des ganzen Leibes abgeschnitten werden muss. Die Leibmetaphorik hat allerdings die verschiedensten Anwendungen. Die Priorität des Ganzen über das Einzelne ist ein wichtiges Anliegen des philosophischen Gebrauchs der Metapher durch die Stoiker. Andere können allerdings die Macht der führenden Teile des Leibes hervorheben oder die Gleichheit aller Glieder. Diese letzte Position für die Gemeinde vertritt z.B. Paulus in 1Kor 12 und Röm 12. Für die komplexe Diskussion über
} 
dass der Körperteil um seinen Erhalt bete, sagt Maximus, weil das einzelne Glied das Wohl des ganzen Leibes wählen müsse und nicht das seinige. Das macht das Gebet unsinnig.

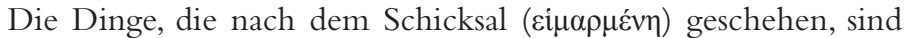
vorbestimmt und können nicht geändert werden. Das Schicksal ist nämlich wie ein Tyrann, der nicht beeinflusst werden kann, sondern stur seinen Willen durchsetzt. Einen König kann man überzeugen, aber keinen Tyrannen. ${ }^{20}$ Es ist deshalb zwecklos, gegen die Bestimmung durch das Schicksal zu beten. Selbst Zeus kann nichts ändern, weil man dem Schicksal nichts als Gegenleistung anbieten kann.

Den Zufall ( irrational, impulsiv und unvorhersehbar ist.

Die Dinge, die aufgrund menschlicher Fertigkeiten ( $\tau \dot{\varepsilon} \chi \vee \eta)$ geschehen, benötigen auch keine Hilfe von den Göttern, sondern einzig das Vertrauen auf die Fähigkeit der Menschen. Ein Handwerker, der sein Produkt anfertigen kann, braucht keine Hilfe von der Gottheit, sondern Selbstvertrauen - wie Seneca auch an Lucilius schrieb.

Diese Beispiele führen Maximus zum Schluss, dass es nicht notwendig ist zu beten. Menschen sollen sich eher auf ihre Fähigkeiten konzentrieren als die Götter um Hilfe anzurufen. In der Argumentation des Maximus kommen viele Perspektiven zusammen, vor allem die stoische Vorstellung, sich am Ganzen auszurichten und dann verantwortlich zu handeln in demjenigen Bereich, der in den eigenen Möglichkeiten liegt. Die Handlung Gottes beschränkt sich

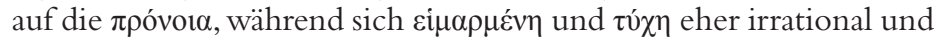
zufällig auswirken. Die $\tau \dot{\varepsilon} \chi v \eta$ fällt in die volle Verantwortung des Menschen. Da sie das konkrete Können des Menschen voraussetzt, ist jeder Rekurs auf die Hilfe Gottes durch Gebet sinnlos. Trotz der kritischen und sarkastischen Einstellung zum Gebet muss Maximus aber zugeben, dass einige der bedeutendsten Philosophen, wie Sokrates, Pythagoras und Plato, öfter gebetet haben. In diesen Formen des Gebets sieht Maximus etwas anderes als im volkstümlichen Bittgebet, weil die Philosophen damit eigentlich einen Dialog mit der Gottheit führen. ${ }^{21}$

Es bleibt in den Ausführungen von Maximus offen, worin die Rolle der Götter besteht. Jedenfalls lassen sich einige Gemeinsam-

die verschiedenen Arten von Leiber und die Anwendung der Leibmetaphorik vgl. L. Scornaienchi, Sarx und Soma bei Paulus. Der Mensch zwischen Destruktivität und Konstruktivität, NTOA 67, Göttingen 2008, 181-207.

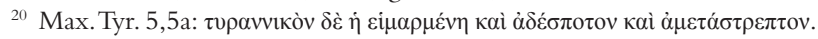

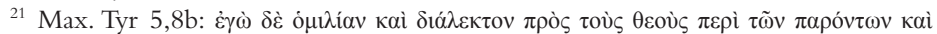

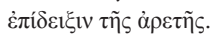


keiten der Vorstellungen des Maximus mit dem Matthäusevangelium feststellen. Sie kommen am besten im Verbot des Schwörens in Mt 5,33-37 zum Ausdruck. Schwören ist verboten, weil jeder mögliche Bezug (Himmel, Erde, oder sogar der eigene Kopf) wiederum auf Gott zurückführt. Das heißt, der Schwörende würde mit einem Bezug auf andere das eigene Tun nicht ernst nehmen, das im Gegenteil im Mittelpunkt steht. Das Schwören ist daher nicht nötig, wenn man sich auf die Wahrhaftigkeit der eigenen Aussage konzent-

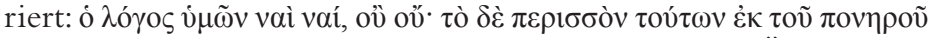
$\dot{\varepsilon} \sigma \tau \imath$ ("Euer Sprechen sei ja, ja und nein, nein und das Überflüssige kommt aus dem Bösen «). Zwischen der Totalität Gottes, dem alles gehört, und dem eigenen Tun, das im Mittelpunkt steht, gewinnt auch die Dimension des Bösen und des Teuflischen Raum, aber als Überflüssiges, als Folge der Ungenauigkeit des eigenen Sprechens. Diese Dimension des Sprechens ist für das Gebet im Matthäusevangelium zentral.

\subsection{Beten aus der Perspektive der Sprachethik}

Die Pointe der Auffassung von Gebet in Mt 6,7-9 ist eine Kritik der Selbstdarstellung beim Beten an Orten, wo sich viele Menschen versammeln und vor allem die Kritik der Formulierung von langen und sich wiederholenden Gebeten. Der Ort spielt zwar eine Rolle, weil das Private als die richtige Einbettung des Gebets deklariert wird, aber das Gebet wird vor allem als Sprechakt verstanden, in dem sich der Glaube und die Frömmigkeit der Betenden wieder-

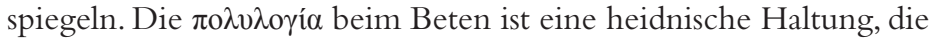
unwirksam ist.

Diese Aufmerksamkeit für das Beten als Sprechakt entspricht dem Interesse des Matthäusevangeliums für das Sprechen, das somit eine ethische Relevanz gewinnt. Dies kann mit der Bezeichnung von W. Baker "Sprachethik «22 oder vielleicht noch genauer "SprachEtikette $«^{23}$ genannt werden. Anders als von Baker vermutet, ist diese

22 Diese Bezeichnung stammt von W. Bakers Untersuchung zum Jakobusbrief (Personal Speech-Ethics in the Epistle of James, WUNT II/68, Tübingen 1995), vgl. v.a. das erste Kapitel "Rudiments of Speech-Ethics«, 23-104. Die Besonderheit der Untersuchung Bakers ist die Thematisierung der Kontrolle des Sprechens, die normalerweise in jeder Kultur zu finden ist. Das Phänomen, das wir im Jakobusbrief oder im Matthäusevangelium beobachten können, ist aber noch speziell innerhalb einer kulturellen Wende in der damaligen Zeit zu verstehen.

${ }^{23}$ Ich neige dazu, dieses Phänomen eine "Sprach-Etikette« zu nennen, weil das Sprechen oft mit dem Problem des Status der Christen und der Wirkung eng verbunden ist. 
besondere Achtung auf die Korrektheit der Sprache nicht nur eine allgemeine Frage aller Epochen. Es ist vielmehr ein Phänomen, das seit der frühen Kaiserzeit die griechisch-römische Kultur interessiert. ${ }^{24}$ Zwei Faktoren scheinen mir dafür prägend: die Einschränkung in der Wortfreiheit seit der Frühkaiserzeit einerseits ${ }^{25}$ und die Auffassung der Philosophie als einer praktischen Kur für das Individuum andererseits, was M. Foucault zu Recht für den Gedanken im 2. Jahrhundert nach Christus als »souci de soi« definiert hat. ${ }^{26}$ Die eingeschränkte Freiheit lässt indirekte Formen der Kommunikation, etwa die satirischen Gattungen, aufkommen, die es ermöglichen, eine gewisse Kritik mit verdeckten Mitteln zu üben. Die »Sorge um das Selbst« qualifiziert die Philosophie als eine praktische Disziplin, die den Menschen hilft, besser zu leben. ${ }^{27}$ Im Neuen Testament wird die Bedeutung der Kontrolle der Sprache mit zwei Absichten verfolgt. ${ }^{28}$ Zum einem will man damit nach außen hin die ethischen Anliegen der Zeit aufnehmen, um die christliche Gemeinde am Diskurs der Zeit partizipieren zu lassen. Das Markusevangelium zeigt einen ruhig argumentierenden Jesus in den Streitgesprächen, was sich von dem sonstigen Bild Jesu als einem Propheten, der eine gewisse Aggression in seiner Argumentation zeigte ${ }^{29}$, abhebt. Zum

${ }^{24}$ Die Definition dieser "Ethik des Sprechens oder des Redens» hat sich im Rahmen meiner Forschung über die Polemik im Neuen Testament als äußerst notwendig erwiesen. Normative Aspekte betreffen auch die am stärksten geführte Attacke, eine Invektive. Vgl. O. Wischmeyer/L. Scornaienchi, Polemik in der frühchristlichen Literatur. Texte und Kontexte, BZNW 170, Berlin/New York 2011. Die normativen Aspekte des Redens und der Argumentation habe ich besonders in meiner Erlanger Habilitationsschrift behandelt: Jesus und seine Apologie. Die Streitgespräche im Markusevangelium.

${ }^{25}$ Die Frühkaiserzeit verzeichnet eine große Einschränkung der Wortfreiheit. Gerade zu dieser Zeit wird die Satire paradoxerweise zur einzigen stilisierten Form der Kritikausübung. Jedoch wird die Satire den Autoren auch oft zum Verhängnis. Vgl. J. Dangel/A. Videau, L'écriture polémique à Rome au début de l'empire, in: G. Declerq u.a. (Hg.), La parole polémique, Colloque, congrès et conférence 11, Paris 2003, 105-130.

${ }^{26}$ M. Foucault, Histoire de la Sexualité, Bd. 3: Le souci de soi, Paris 1984, 57-65.

${ }^{27}$ Interessant in dieser Hinsicht ist die Definition des Denkens Plutarchs als "praktische Ethik", vgl. L. van Hoof, Plutarch's Practical Ethics. The Social Dynamic of Philosophy, Oxford 2010. Plutarch ist auch ein gutes Beispiel eines Autors, der sich sehr mit der Anwendung der Sprache beschäftigt hat. Grundlegend sind drei seiner moralia: "quomodo adulator ab amico internoscatur «; »de recta ratione audiendi«; »de garrulitate«, die alle das Sprechen im Mittelpunkt haben.

${ }^{28}$ Diese Punkte sind Teil meiner aktuellen Untersuchungen und können hier nur schematisch skizziert werden.

${ }^{29}$ Dieses Bild Jesu als Propheten wird m.E. klar in seinen Invektiven in Mt 23. Vgl. L. Scornaienchi, Jesus als Polemiker oder:Wie polemisch darf Jesus sein? Historische 
anderen ist diese Betonung einer Kontrolle der Sprache ein Teil der Polemik nach innen, gegen einige Schriften (vor allem die katholischen Briefe und das Matthäusevangelium ${ }^{30}$ ) und gegenüber dem Werk und der Persönlichkeit des Paulus, weil Paulus als polemischer und aggressiver Theologe in Erinnerung geblieben war.

\section{Das Gebet im Lukasevangelium und in der Apostelgeschichte}

Das lukanische Doppelwerk enthält viele Stellen, die sich indirekt oder direkt mit dem Gebet befassen. Allein schon in der Statistik des

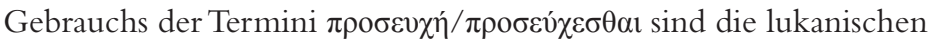
Schriften führend. ${ }^{31}$ Zudem fällt im Vergleich mit den anderen Synoptikern auf, dass Lukas an einigen Stellen im Wirken Jesu eine Gebetshandlung Jesu einführte, die ursprünglich nicht da war. Im lukanischen Bericht der Taufe war Jesus am Beten, als sich die Himmel gespalten haben (Lk 3,21), nach der Speisung der Fünftausend und vor dem Petrusbekenntnis hat er wieder gebetet $(9,18)$ und ebenso vor der Verklärung $(9,28)$ und vor dem Vaterunser $(11,1)$. Er betete auch für Petrus $(22,32)$, für seine Peiniger $(22,34)$ und vor dem Sterben $(23,46) .{ }^{32}$ Die Fülle an Material zum Thema Gebet im Werk des Lukas ist in den letzten Jahren aus verschiedenen Perspektiven untersucht worden. Das Material umfasst Gebete von verschiedenen Menschen, Gebetshandlungen, Gebetstexte und Gebetsparänese. Wie sich das alles in einem Werk einordnen lässt, das eher eine geschichtliche Rekonstruktion des Lebens Jesu und des Anfangs der Kirche ist, bleibt noch offen. Ein wichtiges Thema ist die Bedeutung der Gebetstätigkeit Jesu im dritten Evangelium. Feldkämper definiert die Gebetstexte des Lukas zu Recht als ein "Spannungsbogen"

\footnotetext{
und normative Aspekte, in: Ders./Wischmeyer, Polemik in der frühchristlichen Literatur, 381-413.

${ }^{30}$ Ich teile die Hypothese G. Theißens, dass das Matthäusevangelium in der Bergpredigt eine verdeckte Polemik gegen die paulinische Theologie vertritt.Vgl. G.Theißen, Kritik an Paulus im Matthäusevangelium? Von der Kunst verdeckter Polemik im Urchristentum, in: Wischmeyer/Scornaienchi (Hg.), Polemik in der frühchristlichen Literatur, 465-490.

${ }^{31}$ Im Lukasevangelium begegnen die Termini 19mal, im Matthäusevangelium 17mal; im Markusevangelium 12mal und im Johannesevangelium kein einziges Mal. Lukas

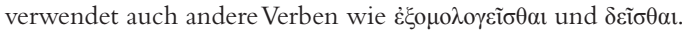

${ }^{32}$ Feldkämper, Der betende Jesus als Heilsmittler, 30, unterscheidet zwei Gruppen von Texten über das Gebet im Lukasevangelium: 1) "Gebetsnotizen« 3,21; 5,16; 6,12, 9,18.28f; 11,1;2) Gebete mit einem Inhalt 10,21f, 22,32.42;23,34.46.
} 
durch das ganze Evangelium. ${ }^{33}$ D. Crump untersucht in seiner Dissertation besonders die Gebetshandlungen Jesu im Lukasevangelium. Er betont den Zusammenhang zwischen dem Beten Jesu und seiner Selbstoffenbarung im Laufe der Ereignisse und vor allem seine Funktion für die Gegenwart der Kirche als Mittler. ${ }^{34}$ Die Gebete Jesu $^{35}$ sind daher nach Crump funktional zur lukanischen Christologie und für die Gegenwart des Lebens der Kirche. Jesus ist einerseits ein Betender wie jeder andere Christ, deshalb dient er als Vorbild, anderseits ist er aber nicht nur ein Betender, sondern er ist auch der einzige Mittler zwischen den Menschen und Gott. ${ }^{36}$ N. Förster behandelt alle Texte zum Gebet im lukanischen Doppelwerk, indem er sie mit paganen und jüdischen Gebetshandlungen vergleicht. Er ist allerdings besonders an den gemeinschaftlichen Gebeten interessiert, die eigentlich nur in der Apostelgeschichte eindeutig zu finden sind. ${ }^{37}$ Försters Untersuchung bietet eine interessante Darstellung des Gebets in der griechisch-römischen Welt und im Judentum.

Mein besonderes Interesse richtet sich hier aber auf die Gebetsparänese des Lukasevangeliums, ${ }^{38}$ die in drei Gleichnissen vorliegt. Das Gleichnis des Freundes um Mitternacht (Lk 11,5-8), das Gleichnis von der Witwe und dem ungerechten Richter $(18,1-8)$ und das Gleichnis vom Pharisäer und Zöllner (18,9-14). Da ich mich für meine Fragestellung auf den Kontrast zwischen Insistieren und sich auf Gott verlassen konzentrieren möchte, lasse ich das Gleichnis vom Pharisäer und Zöllner beiseite. Dieses enthält übrigens eine ähnlich kritische Perspektive wie der matthäische Text: beim Pharisäer, einem Vorbild für die damalige Vorstellung, werden die selbstdarstellerischen Aspekte seiner Frömmigkeit aufgedeckt, während die Haltung des Zöllners, der sich als unwürdig vor Gott stellt, als beispielhaft angesehen wird.

\footnotetext{
${ }^{33}$ Feldkämper, Der betende Jesus als Heilsmittler, 304.

${ }^{34}$ Crump, Jesus the Intercessor, formuliert seine These auf S. 21 wie folgt: »The basic argument of this entire study will be that Luke presents Jesus primarily, though not exclusively, as an Intercessor whose prayers on behalf of the disciples serve to accomplish all that is required for successful, obedient discipleship - including their calling, illumination and perseverance."

${ }^{35}$ Crump benutzt den Ausdruck »Jesus' prayer-life» (z.B. S. 20).

${ }^{36}$ Crump, Jesus the Intercessor, 240f.

${ }^{37}$ Förster, Das gemeinschaftliche Gebet in der Sicht des Lukas, 251-425.

${ }^{38} \mathrm{Zu}$ diesem Thema immer noch grundlegend ist die Studie von Ott, Gebet und Heil, die die Forschung über das Gebet im lukanischen Doppelwerk eröffnet hat.
} 


\subsection{Zu Gott beten wie jemand, der um Mitternacht einen Freund um Hilfe bittet (Lk 11,1-13)}

Die Gleichnisse, die sich im lukanischen Sondergut mit dem Gebet befassen, werden seit der Unterscheidung von Jülicher meistens "Parabeln« genannt. Eine Ausnahme bildet das Gleichnis vom Pharisäer und Zöllner, das Jülicher unter die "Beispielerzählungen" zähltt. ${ }^{39}$ Diese tragen exemplarische Handlungen vor, die unmittelbar für die Paränese angewendet werden sollen. Die Unterscheidung zwischen "Parabel" und "Beispielerzählung" ist aber umstritten. ${ }^{40}$ Gerade im Fall der Gleichnisse des lukanischen Sondergutes scheint es unangemessen, eine zusätzliche Textsorte zu konstruieren. Man kann außerdem eine grundsätzliche Homogenität der eigentlichen lukanischen Gleichnisse feststellen. Sie enthalten nämlich fast immer eine sozial-engagierte Aufforderung oder eine bissige Kritik, die viele Formen annehmen kann wie die konkrete Hilfsbereitschaft eines Samariters gegenüber der Gleichgültigkeit der Geistlichen (Lk 10,20-37), die Frage des Reichtums und der Armut (Lk 12,16-21; Lk 16,19-31) und das Recht einer Witwe (Lk 18,1-8). Ein diskriminierter und meistens unethischer Mensch wie der Zöllner (das Gleiche betrifft den Samariter) kann beispielhaft handeln. Sogar die Handlung eines unehrlichen Verwalters kann in Lk 16,1-8 als konstruktive Anwendung von ungerechtem Reichtum angesehen werden. Es ist deshalb fraglich, ob eine scharfe Unterscheidung zwischen Parabel und Beispielerzählung im Lukasevangelium sinnvoll ist. In

\footnotetext{
${ }^{39}$ A. Jülicher, Die Gleichnisreden Jesu, I. Die Gleichnisreden Jesu im Allgemeinen, Tübingen 1910, 117. Die Parabel wird von Jülicher als »Fabel im Dienst religiöser Ideen« definiert. Eine Unterscheidung allerdings von den "Beispielerzählungen" ist schwierig durchzuführen. Vor allem sind die Beispielerzählungen, die Jülicher unterscheidet, ebenfalls Gleichnisse aus dem lukanischen Sondergut, und zwar vier vom barmherzigen Samariter (Lk 10,29-37), vom Pharisäer und Zöllner (Lk 18,9-14), vom törichten Reichen (Lk 12,16-21) und vom reichen Mann und dem armen Lazarus (Lk 16,19-31), siehe Die Gleichnisreden II, 585-641.

${ }^{40}$ R. Zimmermann, Einleitung, in: Ders. (Hg.), Kompendium der Gleichnisse Jesu, München 2007, stellt die Kategorisierung von Jülicher in Frage. S.19: „Fazit: die Texte, die Jülicher als Beispielerzählungen klassifiziert hat, lassen sich problemlos mit den Kriterien der Parabel beschreiben. "Die unmittelbare Vorbildlichkeit, die Jülicher den Beispielerzählungen zuschreibt, sei aber im Fall des barmherzigen Samariters nicht so klar. Zimmermann macht das Beispiel eventueller jüdischen Rezipienten, die sich kaum mit dem Gleichnis vom barmherzigen Samariter identifizieren können. (S. 18) Das scheint mir nicht das Problem zu sein, weil das lukanische Werk oft eine judenkritische Einstellung hat. Problematisch scheint eher die Trennung im lukanischen Sondergut. Man könnte fast sagen, dass alle Gleichnisse des lukanischen Sondergutes eine Art »Beispielerzählungen« sind.
} 
den Gleichnissen des Sondergutes widerspiegelt sich der Kern des Engagements des Lukasevangeliums, nämlich die Verkündigung Jesu Christi, die die Befreiung des Menschen besagt (Lk 4,18-19) und das Gottesreich vergegenwärtigt. Es ist daher bemerkenswert, dass das Thema "Gebet « in diesen so konnotierten Textsorten behandelt wird. Gebet ist für Lukas immer, nicht nur auf der Ebene der Metaphorik, mit konkreten Handlungen verbunden.

Die Parabel vom bittenden Freund ist in einen Kontext eingebettet, in dem die Diskussion zum Gebet ausführlich zur Sprache kommt. Der Ausgangspunkt in V. 1 ist die Gebetshandlung Jesu und die Bitte eines Jüngers »Herr, lehre uns beten«. Darauf folgt die lukanische Version des Vaterunsers $(11,2 \mathrm{~b}-4)$ und die Parabel $(11,5-8)$. Als Schluss der Rede über das Gebet wird eine Q-Stelle eingefügt über das Bitten (Lk 11,9-11/Mt 7,7-11). Literarisch ist alles sehr gut harmonisiert: Der Text des Vaterunsers ist eine Antwort auf die Frage nach der Unterweisung zum Beten, die Parabel, das Bitten eines Freundes um Mitternacht, eine Illustration der traditionellen Q-Worte.

In der Parabel kommen zwei Perspektiven zum Ausdruck: die Not des Freundes ( $\chi \rho \tilde{\sigma} \sigma o ́ v ~ \mu o \imath)$ draußen, der plötzlich einen Gast bekommen hat, und die Situation der anderen im Haus ( $\check{\varepsilon} \sigma \omega \theta \varepsilon v)$, die um jene Stunde schon am Schlafen sind. Die erste Reaktion ist eine

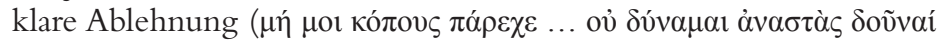
бot). In 11,8 aber kommt es zu einer Umkehr von dieser ersten Ablehnung, die mit einer chiastischen Struktur formuliert wird ${ }^{41}$ :

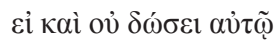

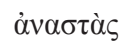

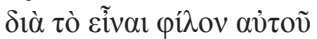

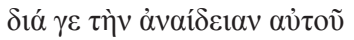

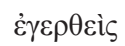

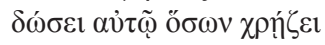

In der Mitte stehen zwei unterschiedliche Begründungen, die Freundschaft einerseits und die Unverschämtheit andererseits, die zwei mögliche Schlüsse der Geschichte präsentieren. Diese zwei Begründungen und Schlüsse stehen in Bezug zu einander in ei-

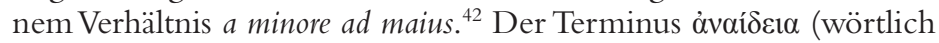

\footnotetext{
${ }^{41}$ M. Wolter, Das Lukasevangelium, HNT 5, Tübingen 2008, 411 f.

42 Ebd., 412, erklärt es, wie folgt: "Selbst wenn der unwahrscheinlichste Fall eintreten sollte, dass er nicht schon um der Freundschaft willen der Bitte nachkommt, so wird ihn doch mit Sicherheit die ảvaíosı seines Freundes dazu bringen, diesem die Brote zu geben".
} 
"Unverschämtheit«, "Dreistigkeit«) ${ }^{43}$ hat eine negative Bedeutung, die nicht zu einem Verhältnis der Freundschaft passen kann. ${ }^{44} \mathrm{~L}$. Schottroff sagt, dass es nicht klar ist, ob diese Unverschämtheit des Freundes gegen die Regel der Freundschaft verstößt oder nicht. ${ }^{45}$ Die Kühnheit der Bitte wird im Allgemeinen negativ bewertet und das hat im Text eine negative Auswirkung. Aber wie oft wird in den lukanischen Gleichnissen ein extremes Verhalten, das in der Regel nicht exemplarisch ist, wider Erwarten zum Beispiel. Die Kühnheit einer solchen Bitte um Mitternacht wird implizit gelobt.

Die folgenden Q-Verse fungieren als eine praktische Anwendung der Parabel. Im Mittelpunkt stehen drei Verbpaare bestehend aus einem Imperativ und dem daraus folgenden Ergebnis im Futur:

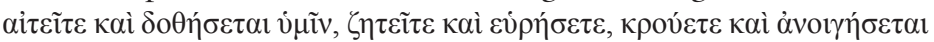

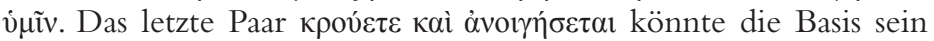
für die lukanische Komposition der Parabel oder steht zumindest im Zusammenhang mit dieser. Die Pragmatik ist jedenfalls klar: Die Adressaten sind aufgefordert, die gleiche Kühnheit des Freundes um Mitternacht anzuwenden, denn sie können nur auf diese Weise erhört werden und bekommen nur so, was sie verlangen.V. $10 \pi \tilde{\alpha} \varsigma$

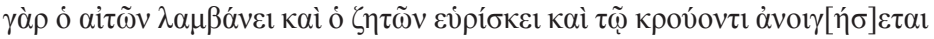
wiederholt die Gewissheit um die Erhörung unserer Bitte.

Die weitere Metaphorik, die auch in Q enthalten ist (Mt 7,9-11/ Lk 11,11-13), führt die Rede eindeutig auf das Gebet zurück und nimmt das Thema des Vaterunsers, die Anrufung "Vater" wieder auf.

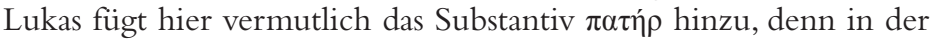
Parallelstelle des Matthäusevangeliums steht nur öv $\theta \rho \omega \pi$ oc. Außerdem beweist dies, dass die syntaktische Form des lukanischen Textes

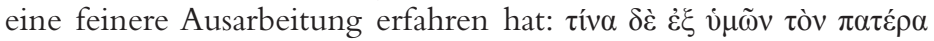

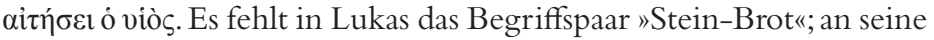
Stelle tritt ein anderes Paar "Ei-Skorpion", was eine merkwürdige Kombination ist. Der Sinn dieser Verse ist schon in Q eine Logik a

\footnotetext{
${ }^{43}$ Vgl. H.G. Liddell/R. Scott, A Greek-English Lexicon (with Supplements), Oxford ${ }^{9} 1996,105$.

${ }^{44}$ A. Merz, Freundschaft verpflichtet (Vom bittenden Freund) Lk 11,5-8, in: Zimmermann (Hg.), Kompendium der Gleichnisse Jesu, 559: „Die Interpretation, die am

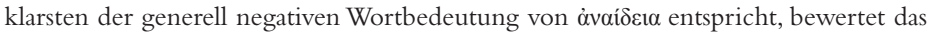
Stören um Mitternacht als schamloses Verhalten, das die Freundschaft über Gebühr strapaziert."

${ }^{45}$ L. Schottroff, Die Gleichnisse Jesu, Gütersloh 2005, 248: "Es ist hier schwierig zu erkennen, ob er damit gegen die gesellschaftlichen Regeln, die Freundschaften definieren, verstößt oder nicht. Der Text will ihn nicht negativ darstellen und macht deutlich, dass er sich nicht wegen der Freundschaft verpflichtet fühlt, aufzustehen und das Brot zu holen, sondern weil er den lästigen Besuch los sein will.»
} 
minore ad maius, die sehr gut zum Gebet passt. Wenn der irdische Vater seinem Sohn etwas Gutes gibt, umso mehr wird der himmlische Vater den Bittenden geben, was sie verlangen. Das höchste Gut, das man von Gott erlangen kann, ist allerdings im lukanischen Text der Heilige Geist. ${ }^{46}$

Es ist interessant zu bemerken, dass diese Q-Verse im Matthäusevangelium zwar in der Bergpredigt vorkommen, aber nicht im Zusammenhang mit dem Gebet und mit dem Vaterunser stehen. Sie sind Teil des Kapitels 7, das eine allgemeine Paränese enthält.

\subsection{Zu Gott beten wie eine Witwe, die einen ungerechten Richter anfleht (Lk 18,1-8)}

Die zweite zu untersuchende Stelle ist die Parabel von der Witwe und dem ungerechten Richter. Der Parellelismus zur Parabel vom bittenden Freund ist eindeutig und wird von den meisten Exegeten anerkannt. ${ }^{47} \mathrm{M}$. Wolter schreibt zum Beispiel dazu: „Die inhaltliche Entsprechung zu 11,5-8 ist offenkundig, obwohl dort nicht die Be-

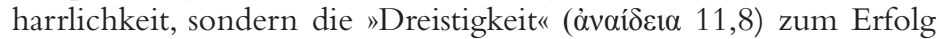
führt. Darüber hinaus basiert die Anwendung in V.7-8 aber auch auf der gleichen Logik wie $11,13 .{ }^{48} \mathrm{~W}$. Ott bemerkt in beiden Parabeln die gleiche Struktur und den gleichen Duktus: Die Situation, die Bewegung, die Bitte und das erste Scheitern. Der Schluss der Parabel ist syntaktisch parallel, in beiden Parabeln finden wir die gleiche

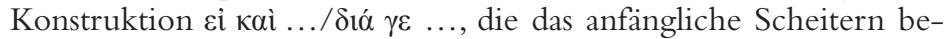
seitigt und überraschenderweise zu einem positiven Schluss führt. ${ }^{49}$

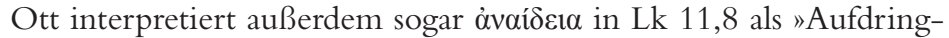
lichkeit des Bittens« und nicht als Unverschämtheit und Frechheit,

\footnotetext{
${ }^{46}$ Schottroff, Gleichnisse, 248f, findet die Nennung des heiligen Geistes einen Hinweis darauf, dass das Beten eine eschatologische Bedeutung hat. Im Geist Gottes verbindet sich jede Erneuerung und jede Gabe des Gottesreiches: „Der Geist Gottes ist die von Gott geschenkte Kraft zur Erneuerung des Lebens nach dem Willen Gottes. Der Geist Gottes macht fähig, Kranke zu heilen, das Evangelium der Armen auszubreiten und vor Gericht mutig zu sein" (249).

${ }^{47}$ Eine Ausnahme ist H. Weder, Die Gleichnisse Jesu als Metaphern. Traditions- und redaktionsgeschichtliche Analysen und Interpretationen, FRLANT 120, Göttingen ${ }^{4}$ 1990. Er schreibt auf S. 270: "Bei der Interpretation dieser Parabel ist besonders darauf zu achten, daß sie nicht von Lk 11,5-8 her ausgelegt wird. Wird dies trotzdem getan - die Texte selbst geben dazu keinerlei Anlaß -, so erhalten die Unterschiede unserer Parabel gegenüber Lk 11,5-8 ein viel zu großes Gewicht«.

48 Wolter, Das Lukasevangelium, 586.

${ }^{49} \mathrm{Ott}$, Gebet und Heil, $13 \mathrm{f}$.
} 
wie es philologisch korrekter ist, und das macht den Parallelismus noch stärker. ${ }^{50}$ Das ist aber m.E. nicht nötig.

Die Parabel stellt einen sozial benachteiligten Menschen in den Mittelpunkt, eine Witwe, die Gerechtigkeit vor einem ungerechten Richter sucht. Die soziale Benachteiligung bestimmt von Anfang an den Misserfolg ihrer Bemühungen. W. Cotter untersucht in einem Aufsatz die sozialen Verhältnisse der Parabel, die eine Rolle beim Verständnis des Textes spielen. ${ }^{51}$ Das Bild der Witwe, die Gerechtigkeit sucht, ist ein Motiv, das oft im Alten Testament vorkommt (Ex 22,22-24; Dtn 10,18; 24,17; Mal 3,5; Jes 54,4 ...). Eine Frau hatte in römischen Rechtsangelegenheiten keinen Zutritt zum Gericht, ${ }^{52}$ obwohl es immer wieder Beispiele von Frauen gab, die solche sozialen Konventionen brachen und sich als Heldinnen erwiesen oder aber für ihre Unverschämtheit getadelt wurden. ${ }^{53}$ Der Bruch der sozialen Konventionen durch die Witwe in der Parabel bringt die Beharrlichkeit der Frau viel näher an die Unverschämtheit des bittenden Freundes um Mitternacht heran. Das andere Element im Gleichnis ist die Charakterisierung des Richters, der in Lk 18,1

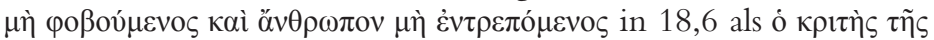

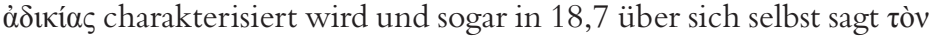

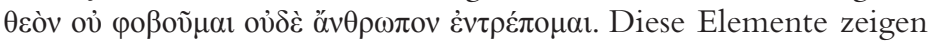
ein eindeutiges Bild von einem Menschen, von dem keine Gerechtigkeit zu erwarten ist, schon gar nicht, wenn eine Frau darum bittet. Der Richter steht allerdings auf einer Ebene mit Gott. Er erreicht vielleicht gerade durch seine extrem negativen Eigenschaften eine Position der Distanz von den Normen und von den Menschen, die eigentlich charakteristisch für Gott ist. ${ }^{54}$ Er bleibt aber ein Richter

\footnotetext{
${ }^{50}$ Ott, Gebet und Heil, 30.

${ }^{51}$ W. Cotter, The Parable of the Feisty Widow and the Threatened Judge (Luke 18.18), NTS 51(2005), 328-343.

${ }^{52}$ Cotter, The Parable of the Feisty Widow, 333: »In the Mediterranean world a modest woman did not visit the public offices of the court [...] Women's inatural condition belonged in the domestic, private sphere of the home, not in public male domain of the courts, and any woman who frequented public male space would be seen to be inviting male attention and violating sthe modesty of the matron's rober." ${ }^{53}$ Ein Beispiel ist Carfania, Frau eines Senators, die ihre Rechtssachen selbst vertrat. Dazu die Kritik von Valerius Maximus 8,3.1 und von Juvenal, sat. 6,242-245 "origo vero introducta est a Carfania improbissima femina, quae inverecunde postulans et magistratum inquietans causam dedit edicto", Zitat aus Cotter, The Parable of Feisty Widow, 335, Anm. 30.

${ }^{54}$ Weder, Die Gleichnisse Jesu, 270, wendet den Ausdruck "sans loi ni foi« an, um den Richter zu charakterisieren. Ein interessanter Aspekt ist Weders Definition von der "Selbstherrlichkeit» des Richters. Der Richter sei in seiner Ungerechtigkeit über das Gesetz erhaben, sei in der Position Gottes, der nicht an die Menschen und an Gesetze
} 
der Ungerechtigkeit, wie die irdischen Väter in Lk 11 im Grunde trotz ihrer Vaterschaft »böse« bleiben. Der ganze Text ist voller Begriffe, die die Gerechtigkeit bzw. die Ungerechtigkeit betonen. Das besagt, dass das Gebet im Lukasevangelium mit der konkreten Frage nach der Gerechtigkeit verbunden ist, und dies nicht nur auf einer metaphorischen Ebene. Auf der Basis des Textes kann man allerdings den Fall der Frau nicht genauer bestimmen, wie es L. Schottroff versucht: sie sei "Opfer eines Mannes, der ihre wirtschaftliche Lebensgrundlage angetastet hat.$^{55}$

Die Umkehr des anfänglichen Misserfolgs geschieht unerwartet. Die Parabel referiert eine tiefe Überlegung des Richters, der sich zu seiner Ungerechtigkeit bekennt, der aber der Bitte der Witwe

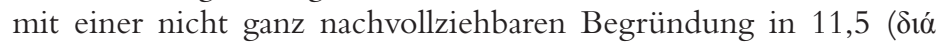

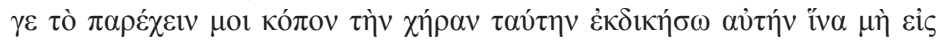

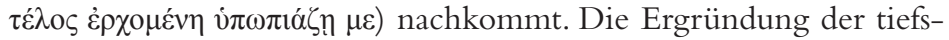
ten Gedanken der Gestalten der Gleichnisse und deren sprachliche Wiedergabe ist eine Charakteristik der lukanischen Gleichnisse und ist m.E. ein Zeichen der lukanischen Echtheit dieser Texte. Ein Argument der Überlegungen des Richters ist die Belästigung durch das ständige Bitten der Frau, das andere Argument ist hingegen nicht unmittelbar verständlich und daher umstritten. Es wird mit

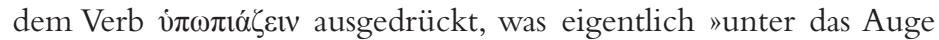
schlagen", "ein blaues Auge machen " heißt. ${ }^{56}$ Es scheint schwierig, dass ein Richter Angst haben könnte, von einer Frau ins Gesicht geschlagen $\mathrm{zu}$ werden, obwohl viele Autoren für diese wörtliche Interpretation plädieren. ${ }^{57}$ Eine metaphorische Deutung scheint

gebunden ist. Das rückt diesen ungerechten Richter näher zu Gott. Was eigentlich fast unfassbar wäre - der Vergleich von Gott mit einem ungerechten Richter - realisiert sich faktisch in dem Extrem der Gesetzeslosigkeit. Auch A. Merz, Die Stärke der Schwachen (von bittenden Witwen) Lk 18,1-8, in: R. Zimmermann, Kompendium der Gleichnisse Jesu, 667-680, 667. „Die wahrscheinlich am heftigsten umstrittene Frage ist, ob der Richter im Gleichnis als eine auf Gott hin transparente Figur fungiert oder nicht. Ausgehend von dieser verschieden zu beantwortenden Grundfrage lassen sich Deutungstypen der Parabel unterscheiden. [...] Auf der Ebene des Lukasevangeliums ist die Antwort eindeutig, der schließlich doch aktiv werdende Richter und Gott stehen in einem - allerdings komplexen - Entsprechungsverhältnis zueinander."Vgl. Die Diskussion auch bei Cotter, The Parable of Feisty Widow, 330. L. Schottroff, Die Gleichnisse Jesu, 250-251, vertritt hingegen eine Opposition der Gestalt des Richters zu Gott.

${ }^{55}$ Schottroff, Die Gleichnisse Jesu, 251.

${ }^{56}$ Vgl. Liddell \& Scott, Lexicon, S. 1904, "strike under the eye«, "give him a black eye». ${ }^{57}$ Vgl. z.B. Schottroff, Die Gleichnisse Jesu, 253. "Das Selbstgespräch des Richters spiegelt ihre Grenzüberschreitung: Dieser Frau ist es sogar zuzutrauen, dass sie ihn ge- 
mir an dieser Stelle logischer, die eine Steigerung von "belästigen" bedeuten könnte. ${ }^{58}$

Die Anwendung der Parabel ist in 11,1 das beharrliche, unermüdliche Beten und in 11,7 ein Schluss a minore ad maius: umso mehr wird Gott den Auserwählten zu ihrem Recht verhelfen. Gott ist hier als nachsichtig beschrieben. ${ }^{59}$ Der Text scheint mir kohärent, und es sind keine unterschiedlichen Überlieferungen feststellbar.

Die Beharrlichkeit der Witwe ist gemäß Lukasevangelium ein Vorbild für das Beten der Christen. In der Zeit vor der Parusie ist Beten das, was der Gemeinde helfen kann, Versuchungen und Anfechtungen zu überwinden. ${ }^{60}$ Dieses pausenlose Beten ist aber nicht eine Art fatigare deos, wie bei den paganen Religionen. Die Suche nach der Gerechtigkeit ist eine konkrete Sache, die der Frömmigkeit immer einen konkreten Inhalt verleiht.

\section{Insistieren oder sich auf Gott verlassen}

Insistieren oder sich auf Gott verlassen? Das Matthäus- und das Lukasevangelium vertreten diese unterschiedlichen Perspektiven, die auch immer wieder in der Praxis der Kirche und im Leben der Christen als Optionen in einem gegensätzlichen Verhältnis stehen. Gebetspraxis geschieht in konkreten Situationen und darf nicht zu einer abstrakten Theorie werden.

Die Basis beider Evangelien ist gemeinsam: Beide sind in einer klaren Kritik der heidnischen und jüdischen Frömmigkeitspraxis fundiert. Die vielen Worte oder das Selbstlob des Pharisäers müssen

walttätig ins Gesicht schlägt."Schottroff meint, es sei kein metaphorischer Gebrauch belegt.

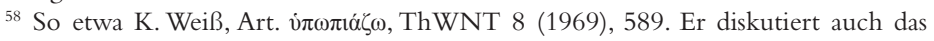

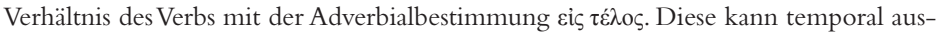
gelegt werden, "am Ende", "schließlich" oder als Bezeichnung des Maßes "gänzlich", "völligu. Der Ausdruck besagt dann entweder "damit sie mich nicht schließlich noch in einem öffentlich Auftritt bloßstellt« oder "damit sie mich nicht durch ihr beharrliches Auftreten völlig aufreibt«.

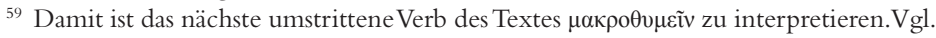
Ott, Gebet und Heil, 46, und Schottroff, Die Gleichnisse Jesu, 255.

${ }^{60} \mathrm{Vgl}$. Weder, Die Gleichnisse Jesu, 272. Diese These wird vor allem von Ott, Gebet und Heil, z.B. 138 ff, vertreten, der die Situation der Kirche vor der verzögerten Parusie in den Mittelpunkt dieser lukanischen Behandlung des Gebets stellt. »Der Gemeinde, die sich weiterhin für unbestimmt lange Zeit in dieser Welt einrichten muss, droht die Gefahr, über den Freuden dieser Welt und den Sorgen um das tägliche Leben $[\ldots]$ den eigentlichen Sinn und das eigentliche Ziel ihres Lebens zu vergessen, bzw. die Gefahr, daß ihr Glaube erstickt wird.« (138) 
vermieden werden. Beide Evangelien stellen die Vorstellung Gottes als Vater in den Mittelpunkt, eine Vorstellung, die klar im Vaterunser zum Ausdruck kommt. Beide Evangelien bezeugen die Worte Jesu der Q-Tradition, dass man beten soll. Für Lukas ist dieses Wort eigentlich die Basis für das Verständnis des Gebets; für Matthäus stehen diese Worte in einem Zusammenhang allgemeiner Paränese. Matthäus versteht das Gebet durch seine Beachtung einer Ethik des Sprechens als Kontrast zu den langen und unverständlichen Gebeten; man soll auf das Sprechen achten im Umgang mit Menschen und auch im Umgang mit Gott. Lukas verbindet das Beten mit der Suche nach Gerechtigkeit in einer ungerechten Welt. Beten hilft, die Gerechtigkeit auch in unmöglichen Situationen zu realisieren. Das sind zwei unterschiedliche Perspektiven. Ist eine Synthese möglich? Vielleicht öffnen sich diese Perspektiven auch heute für die Christen unserer Zeit als Potentialität, ohne eine logische Synthese zu benötigen. 